\title{
Sensorless Robot Collision Detection and Hybrid Force/Motion Control*
}

\author{
Alessandro De Luca Raffaella Mattone \\ Dipartimento di Informatica e Sistemistica \\ Università di Roma "La Sapienza' \\ Via Eudossiana 18, 00184 Roma, Italy \\ \{deluca,mattone\}@dis.uniromal.it
}

\begin{abstract}
We consider the problem of real-time detection of collisions between a robot manipulator and obstacles of unknown geometry and location in the environment without the use of extra sensors. The idea is to handle a collision at a generic point along the robot as a fault of its actuating system. A previously developed dynamic FDI (fault detection and isolation) technique is used, which does not require acceleration or force measurements. The actual robot link that has collided can also be identified. Once contact has been detected, it is possible to switch to a suitably defined hybrid force/motion controller that enables to keep the contact, while sliding on the obstacle, and to regulate the interaction force. Simulation results are shown for a two-link planar robot.
\end{abstract}

Index Terms - Collision detection, fault detection and isolation, hybrid force/motion control.

\section{INTRODUCTION}

When a robot manipulator operates in an unstructured environment or shares its workspace with a human user, safety issues are of primary concern [1]. Main injuries may occur from an accidential collision between the robot structure and the environment (viz. humans), due to the uncertain location of obstacles and/or unpredicted relative motion. Avoiding such collisions requires (at least local) knowledge of the environment geometry and the use of computationally intensive motion planning techniques, see e.g. [2]. Anticipating incipient collisions or detecting them in real-time is typically based on the use of additional external sensors, such as sensitive skins [3], on-board vision [4], strain gauges [5], force load cells, and so on. When an impact occurs, the resulting contact forces may be alleviated, by pursuing a lightweight robot design [6], possibly with distributed compliant characteristics in the driving system and/or through a soft covering of the links [7]. Once a collision is detected, the controller should switch strategy and either stop robot motion or perform a more sophisticated interaction task.

It is obviously more cost effective to be able to detect contacts without the need of additional sensors. Comparing the applied torque (or, the current in an electrical drive) with the nominal model-based command (i.e., the torque expected in the absence of collision) and looking for fast transients so as to detect possible collision is a rather intuitive scheme, see, e.g., [8], [9], [10], which has been refined lately by including an adaptive control scheme [11],

*Work supported by the EU project EU-IST-2001-32122 IFATIS.
[12]. However, tuning of collision detection thresholds in such schemes is difficult because of the highly varying dynamic characteristics of the commanded torques. In addition, a common drawback (even when robot dynamics is perfectly known) is that the on-line torque computation based on inverse dynamics requires acceleration measurements, which introduces in practice noise (due to numerical differentiation of velocity or position data) and/or an intrinsic one-step delay in a digital implementation.

In this framework, we consider the following specific problem.

1) Detect a collision of the robot with an unknown environment, using only the standard proprioceptive sensors (joint encoders). Collision may occur at any point along the robot arm.

2) Switch and execute an interaction control task that requires keeping the contact during motion while regulating force at the contact point. This should be performed without any force sensor.

The main idea that we pursue is to handle the collision as a faulty behavior of the robot actuating system. In fact, the dynamic effect of a cartesian contact force is that of an additional joint torque with respect to the commanded one. Therefore, robot actuator fault detection and isolation (FDI) techniques [13], [14], [15] can be used. These do not require acceleration measurements nor inversion of the robot inertia matrix. In particular, the FDI method based on generalized momenta [13] works independently of the generation scheme for the nominal torque, which may thus be any open-loop command or feedback law. This is particularly convenient when it is necessary to switch control strategy. The FDI scheme produces a residual vector which is a filtered version of the joint torques resulting from cartesian contact forces. Due to an intrinsically decoupled structure, the components of this residual vector contain as much information as possible on the location of the collision force. Furthermore, during the interaction task, the residual vector is used to decompose the joint velocity space into complementary directions, so that a hybrid force/motion controller can be designed. In order to illustrate the performance of the whole approach, simulation results are presented for a two-link planar robot colliding and then sliding along a compliant obstacle. 


\section{SENSORLESS COLLISION DETECTION}

Consider a rigid robot manipulator having $n$ (rotational) joints, with associated generalized coordinates $q$, that may undergo a possible contact with the environment at a generic point of the structure. Using a Lagrangian approach, the robot dynamic model is

$$
M(q) \ddot{q}+c(q, \dot{q})+g(q)=\tau+\tau_{c},
$$

where $M(q)>0$ is the (symmetric) inertia matrix, $c(q, \dot{q})$ is the Coriolis and centrifugal vector, $g(q)$ is the gravity vector, $\tau$ is the commanded joint torque, and

$$
\tau_{c}=J_{c}^{T}(q) F_{c},
$$

is the joint torque associated to a generalized contact force $F_{c}$. The Jacobian matrix $J_{c}(q)$ relates the linear and angular velocity of a frame $\Sigma_{c}$ located at the contact point $P_{c}$ to the joint velocity $\dot{q}$. Both terms in the right-hand-side of eq. (2) are supposed to be unknown.

The generalized momentum $p=M(q) \dot{q}$ associated to the mechanical system (1) satisfies the first-order equation

$$
\dot{p}=\tau+\tau_{c}-\alpha(q, \dot{q}),
$$

where the components of $\alpha$ are given by [13]

$$
\alpha_{i}=g_{i}(q)-\frac{1}{2} \dot{q}^{T} \frac{\partial M(q)}{\partial q_{i}} \dot{q}, \quad i=1, \ldots, n .
$$

Define the residual vector $r$ as

$$
r=K\left[\int(\alpha-\tau-r) d t+p\right]
$$

with (diagonal) $K>0$. The residual dynamics satisfies

$$
\dot{r}=-K r+K \tau_{c}, \quad r(0)=0,
$$

namely that of a linear exponentially stable system driven by the (joint) contact torque $\tau_{c}$. Actually, for every component of the residual dynamics we can write a transfer function

$$
\frac{r_{i}(s)}{\tau_{c, i}(s)}=\frac{K_{i}}{s+K_{i}}, \quad i=1, \ldots, n,
$$

having unitary gain. Note that eq. (7) is similar to the result obtained in [8], where dynamic interaction torques between robot joints, and thus the acceleration $\ddot{q}$, are assumed to be available. Instead, in order to be implemented, eq. (5) requires proprioceptive measures $(q, \dot{q})$ only, the knowledge of the current commanded input $u$, but no acceleration $\ddot{q}$ or inversion of the inertia matrix $M(q)$. For manipulators with many d.o.f., the computation of the generalized momenta $p$ and vector $\alpha$ needed for the implementation of the residual $r$ in eq. (5) may need special care, e.g., by using recursive schemes. A finite difference numerical approximation can also be adopted for the inertial part in eq. (4), see [15].

During free motion, all residuals are practically zero. The rising of one or more residuals above a fixed threshold corresponds to the occurrence of a collision. In particular, for large values of $K_{i}$ in eqs. (5-7), the evolution of $r_{i}$ will reproduce accurately the evolution of the contact torque $\tau_{c, i}=J_{c i}^{T}(q) F_{c}$, being $J_{c i}$ the $i$-th column of

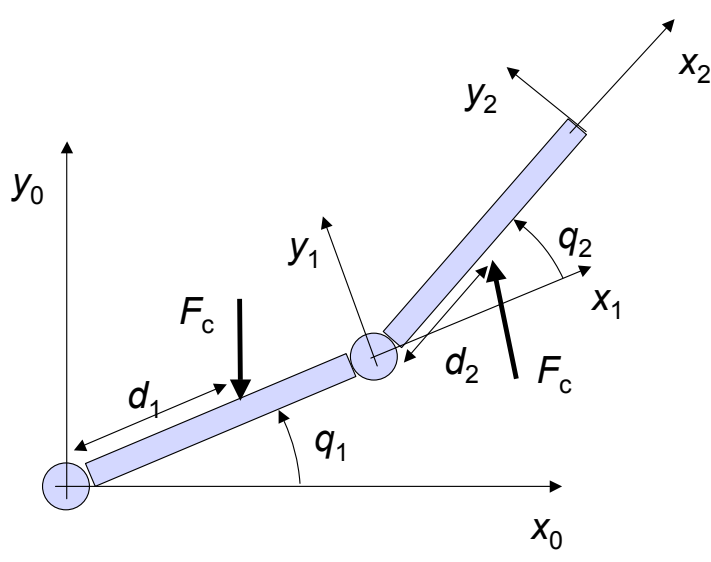

Fig. 1. Possible collision forces in a two-link planar robot

the Jacobian matrix $J_{c}$. Residuals rapidly return to zero as contact is lost. As a result, this FDI-based scheme allows the efficient detection of any robot collision without external sensors. We note that the need of an accurate robot dynamic model can be relaxed by developing an adaptive version of the scheme. Moreover, a dynamic thresholding of residuals can be used so as to avoid false detection due to noise/additional disturbances [16].

\section{IDENTIFICATION OF CONTACT FORCES}

Using our residual generator, it is immediate to identify the robot link that has collided. In fact, assuming that the robot is an open kinematic chain, if a collision occurs on link $k$ it is

$$
r_{i}(t) \neq 0, i=1, \ldots, k, \quad r_{j}(t)=0, i=k+1, \ldots, n,
$$

for the time interval of contact. Indeed, residuals will be affected only by contact forces that perform work on robot motion (not by those balanced by reaction forces in the robot structure, i.e., belonging to the kernel of $J_{c}^{T}(q)$ ). While the distal residuals (i.e., beyond link $k$ ) will certainly be unaffected, the sensitivity to cartesian generalized forces $F_{c}$ of each proximal residual will vary in general with the robot configuration (see also [17]).

By discussing a simple case in 2D, we show next how to extract the largest possible information on impact location and amplitude of the contact force in the case of a single pointwise collision. The following analysis is the dynamic counterpart of the static transformation from cartesian forces to joint torques.

With reference to the two-link planar robot of Fig. 1, a collision force $F_{c}=\left(F_{c x}, F_{c y}\right)$ may arise at point $P_{c 1}$ on the first link, at a distance $d_{1}$ from the first joint, or at point $P_{c 2}$ on the second link, at a distance $d_{2}$ from the second joint. In the first situation, it is

$$
\tau_{c 1}=J_{c 1}^{T}(q) F_{c}=J_{c 1}^{T}(q){ }^{0} R_{1}\left(q_{1}\right){ }^{1} F_{c}=\left[\begin{array}{c}
d_{1}{ }^{1} F_{c y} \\
0
\end{array}\right],
$$


where ${ }^{0} R_{i}$ is the rotation matrix from the base frame to the $i$-th link frame. Equations (6) and (9) show that $r_{2}$ will be unaffected (as expected) while $r_{1}$ will be driven only by the force component normal to the link (scaled by $d_{1}$ ). It is clear that exact location and amplitude of the contact force cannot be identified separately.

In the second situation, it is

$$
\begin{aligned}
\tau_{c 2} & =J_{c 2}^{T}(q) F_{c}=J_{c 2}^{T}(q){ }^{0} R_{2}\left(q_{1}, q_{2}\right)^{2} F_{c} \\
& =\left[\begin{array}{c}
l_{1} s_{2}{ }^{2} F_{c x}+\left(l_{1} c_{2}+d_{2}\right)^{2} F_{c y} \\
d_{2}{ }^{2} F_{c y}
\end{array}\right],
\end{aligned}
$$

and $r_{2}$ will always be affected only by the force normal to the link (scaled by $d_{2}$ ), while $r_{1}$ will be excited in general by both the normal and the tangential components of the contact force.

Equations (9-10) contain the largest information that can be extracted from residual behavior, without external sensing. Additional knowledge about the collision characteristics may be used for further identification. For example, if we assume that collision may occur only at the robot end-effector, then $d_{2}=l_{2}$ in eq. (10) is known and the two components $\left({ }^{2} F_{c x},{ }^{2} F_{c y}\right)$ can be generically identified from eqs. (6) and (10). Alternatively, if the obstacles have smooth contours and the collision point is 'internal' to the second link, then ${ }^{2} F_{c x} \approx 0$ (the link is tangential to the obstacle) and eqs. (6) and (10) can be used to identify both $d_{2} \in\left(0, l_{2}\right)$ and ${ }^{2} F_{c y}$.

\section{HYBRID FORCE/MOTION CONTROL}

In this section we show how to exploit the information on the contact force provided by the residual vector (5) in order to devise a hybrid force/motion control scheme that ensures motion of the contact point along the obstacle surface and approximate regulation of the contact force.

As shown in Sect. III, the exact contact location and the associated cartesian force cannot be independently identified in general. However, hybrid tasks can be accomplished by suitably processing at the joint-space level the dynamic effects of the robot-environment interaction. Performance of the proposed control scheme will indeed depend on the accuracy of the joint contact torque 'measure' provided by the residual $r$, i.e., on the accuracy of the model and on the input and measurement noise affecting the system. Moreover, the bandwidth of the low-pass filters in eq. (7) should be larger than that of the force and motion reference signals. On the other hand, control tasks of practical interest for robots in contact with unstructured environments are usually performed at low velocities and require only a rough regulation of interaction forces.

We consider only obstacles with smooth surfaces and at most one pointwise contact between the robot and the environment. Without loss of generality, we can take $K_{i}=K, i=1, \ldots, n$, in eq. (7), so that vectors $\tau_{c}$ and $r$ will have the same direction in $\mathbb{R}^{n}$ even during transients. Finally, we assume that friction at the contact is negligible, i.e., the contact force is directed along the normal to the obstacle surface.
In standard hybrid force/motion control schemes, the task space is decomposed into complementary directions, where only force and, respectively, motion is controlled [18], [19]. Here, such decomposition is locally performed in the joint velocity space, since the location and orientation of the cartesian task frame $\Sigma_{c}$ is not known. In particular, it is

$$
\tau_{c}=\frac{r}{\|r\|} f=\hat{r} \cdot f
$$

where $f=\left\|\tau_{c}\right\|$. Moreover, the velocity of the contact point between robot and environment is assumed to be tangent to the obstacle surface ${ }^{1}$. As a result, the joint velocity vector $\dot{q}$ during contact can be locally parametrized as

$$
\dot{q}=T s,
$$

where $s \in \mathbb{R}^{n-1}$ and $T$ is a $n \times(n-1)$ matrix, whose columns (normalized to unity) span the $(n-1)$-dimensional subspace orthogonal to $r$, i.e., such that $r^{T} T=0$. In fact, although the location of the contact point on the robotic structure is unknown, a motion of the cartesian contact point along the obstacle surface corresponds to joint displacements in the null space of $r^{T}$ (by the principle of virtual work). Thus, the joint accelerations $\ddot{q}$ can be written as

$$
\ddot{q}=T \dot{s}+\dot{T} s \approx T \dot{s},
$$

where the last approximation is valid for 'low velocity' motion along the smooth surface of the obstacle. Substituting eqs. (11) and (13) into model (1) yields

$$
\left[\begin{array}{ll}
M(q) T & -\hat{r}
\end{array}\right]\left[\begin{array}{c}
\dot{s} \\
f
\end{array}\right]+c(q, \dot{q})+g(q)=\tau,
$$

where the $n \times n$ matrix $\mathcal{M}(q)=[M(q) T-\hat{r}]$ is nonsingular by construction. Then, the nonlinear feedback control law

$$
\tau=\mathcal{M}(q) u+c(q, \dot{q})+g(q)
$$

results in the linear and decoupled dynamics

$$
\left[\begin{array}{l}
\dot{s} \\
f
\end{array}\right]=u=\left[\begin{array}{l}
u_{s} \\
u_{f}
\end{array}\right] .
$$

As a consequence, motion tracking for $s$ and force regulation for $f$ can be executed by the linear control laws

$$
\begin{aligned}
& u_{s}=\dot{s}_{d}+K_{P s}\left(s_{d}-s\right)+K_{I s} \int\left(s_{d}-s\right) d t, \\
& u_{f}=f_{d}+\left(K_{P f}-1\right)\left(f_{d}-f\right)+K_{I f} \int\left(f_{d}-f\right) d t,
\end{aligned}
$$

for positive gains $K_{P s}, K_{I s}, K_{P f}$, and $K_{I f}$, and where $s_{d} \in \mathbb{R}^{n-1}$ and $f_{d} \in \mathbb{R}$ denote the desired (reference) behaviors for $s$ and $f$, respectively. As concerns the 'measure' of the velocity and force parameters $s$ and $f$ to be used in (17), according to the assumptions made in this section, we can take $f=\|r\|$ and $s=T^{\#} \dot{q}=\left(T^{T} T\right)^{-1} T^{T} \dot{q}$.

\footnotetext{
${ }^{1}$ In case of a compliant contact, the velocity in the normal direction is still negligible for a quasi-static interaction between robot and obstacle.
} 


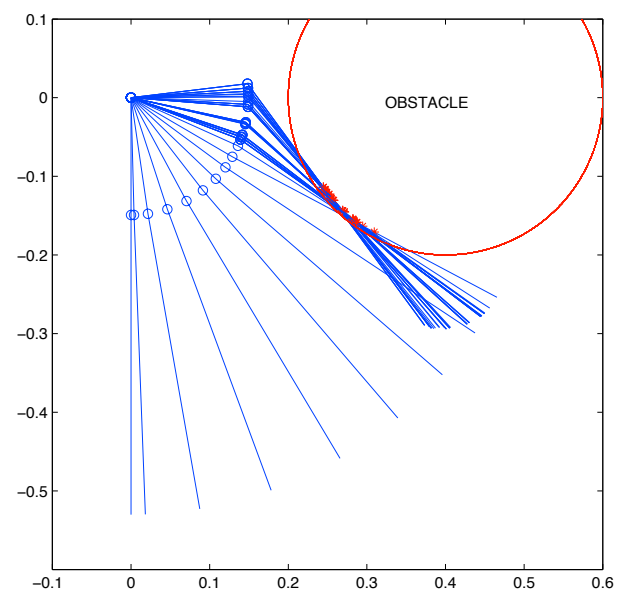

Fig. 2. Stroboscopic view of the robot during free and contact motion (contact points are identified by stars)

\section{Simulation RESUlTS}

In order to test the proposed collision detection and hybrid force/motion control scheme, we have performed numerical simulations for a $2 R$ robot under gravity ${ }^{2}$. The joint positions $\left(q_{1}, q_{2}\right)$ are assumed to be measured by digital encoders (with resolutions $\pi / 4096$ and, respectively, $\pi / 2048$ [rad]), while joint velocities are obtained by numerical differentiation. This measurement process introduces a realistic level of noise in the simulation.

During free motion, the robot is being regulated to $q_{d}=$ $(\pi / 2,0)(q=(0,0)$ is the downward free equilibrium configuration) by a decoupling and linearizing state feedback law (computed torque). At time $t_{c}$, the second link collides with a circular obstacle of radius $\rho=0.2[\mathrm{~m}]$ and centered at $C=(0.4,0)[\mathrm{m}]$ (see Fig. 2). The stiffness coefficient of the robot-obstacle compliant contact is $K_{c}=50[\mathrm{~N} / \mathrm{m}]$.

The behaviors of the residual $r$ and of the joint velocity $\dot{q}$ are shown in Figs. 3 and 4, respectively. Collision is detected when at least one of the residual components exceeds the threshold value 0.02 . This time instant $\left(t_{c}=0.795[\mathrm{~s}]\right)$ is indicated by the first vertical line shown in the figures. The second vertical line corresponds to the time instant where the controller is switched to the hybrid force/motion law (15-17). This event is triggered at $t_{s w}=4.133$ [s], after the norm of the joint velocity has been constantly below a fixed threshold $\left(v_{\text {thres }}=0.015[\mathrm{rad} / \mathrm{s}]\right)$ for a given time $(\Delta t=0.175[\mathrm{~s}])$, i.e., when the assumption of quasistatic interaction can be considered valid. Note that, under regulation by computed torque control and for a compliant contact, this condition is certainly attained in finite time. In general, however, the transient behavior of contact forces and joint velocities might result unsatisfactory just after collision, thus requiring a suitable stability analysis and control reconfiguration for the post-impact phase (not

\footnotetext{
${ }^{2}$ The two links of the robot are thin rods with masses $m_{1}=0.193$ and $m_{2}=0.073[\mathrm{~kg}]$, lengths $l_{1}=0.1492$ and $l_{2}=0.381[\mathrm{~m}]$, and inertias $I_{1}=0.0015$ and $I_{2}=1.949 \cdot 10^{-4}\left[\mathrm{kgm}^{2}\right]$. The link centers of mass are located on their axes, at a distance $h_{1}=0.1032$ and, respectively, $h_{2}=0.084[\mathrm{~m}]$ from the joints.
}

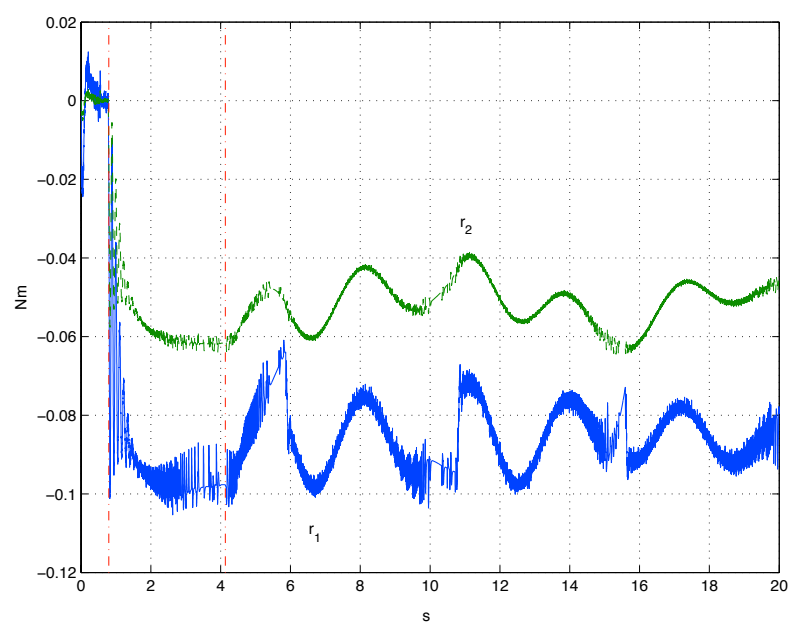

Fig. 3. Residuals (free motion, collision, and hybrid control phases are separated by vertical dash-dotted lines)

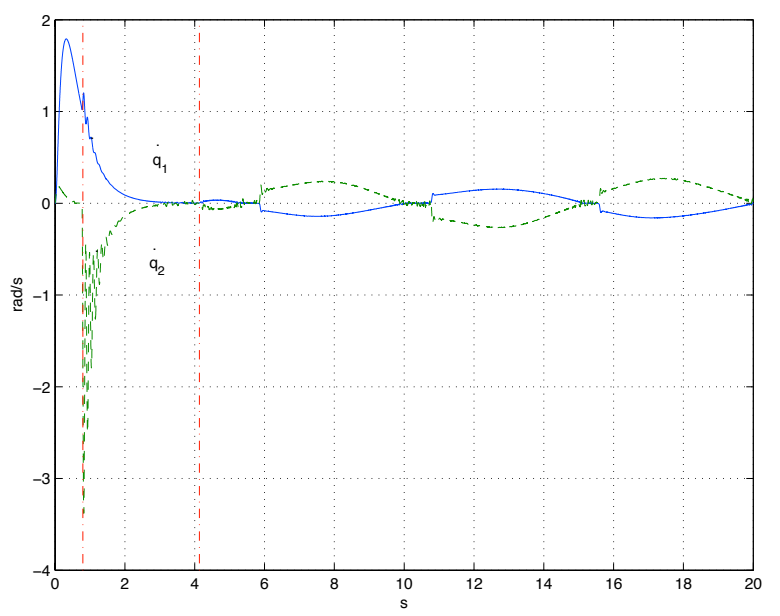

Fig. 4. Joint velocities

needed in the present numerical case).

The (joint-space) control objectives of the hybrid force/motion task are the regulation of $f=\left\|\tau_{c}\right\|$ to the desired value $f_{d}=0.1[\mathrm{Nm}]$, and the tracking of the trajectory $s_{d}(t)=0.2 \sin 0.2 \pi t[\mathrm{rad} / \mathrm{s}]$ for the parameter $s$ in eq. (12). The control gains in eq. (17) are $K_{P s}=2$, $K_{I s}=1, K_{P f}=0.01$, and $K_{I f}=0.1$. Moreover, $K_{1}=K_{2}=50$ in eq. (5). The resulting behavior of the controlled variables $f$ and $s$ are reported in Figs. 5 and 6 , respectively. It can be seen that there is a good tracking of the periodic motion, while the mean value of the joint contact torque norm stabilizes at the desired level (remember that the hybrid task starts only after $t_{s w}$ ).

Although the hybrid control objectives can be imposed only at the joint-space level, one is indeed interested in the cartesian force/motion behavior at the contact. Figure 7 shows the actual cartesian normal force at the contact point (which is unknown to the controller), while the angular position of the contact point on the circular obstacle is given in Fig. 8. Again, the periodic motion along the obstacle tangent is performed at the correct frequency (with 


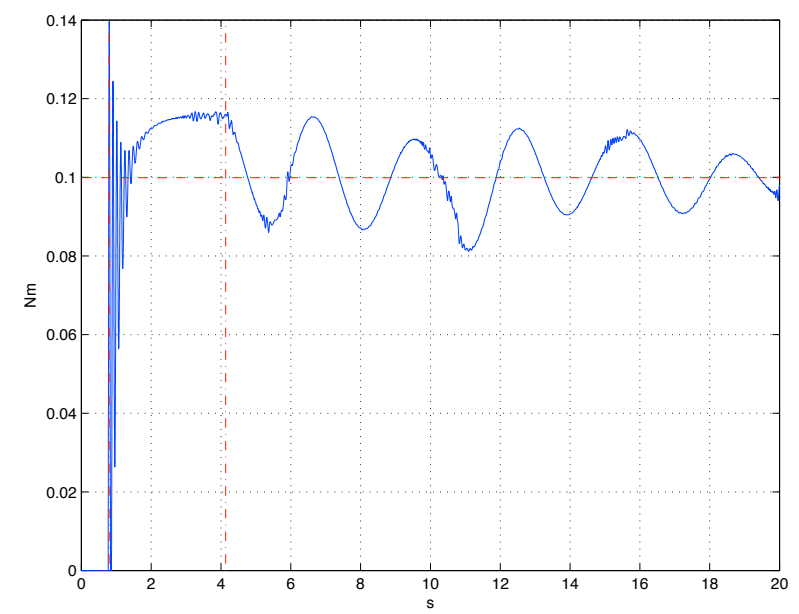

Fig. 5. Actual (blue, solid) and desired (red, dashed) norm $f=\left\|\tau_{c}\right\|$ of contact torques at the joints

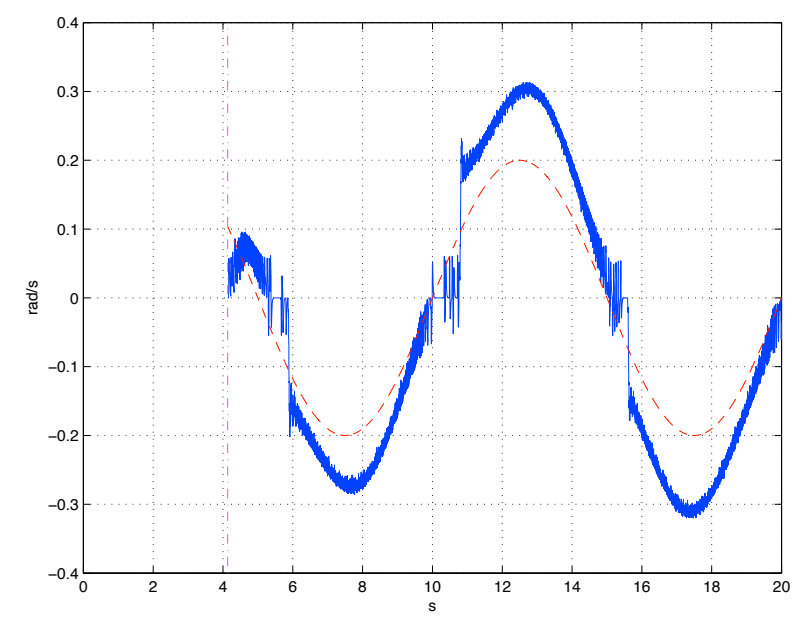

Fig. 6. Actual (blue, solid) and desired (red, dashed) behavior of parameter $s$ (amplitude of joint velocity along the generalized contact surface - see eq. (12))

an excursion of about $2 \pi \rho \cdot(19 / 360)$ [m] on the contour), while there is a sufficient limitation on the cartesian normal force (the peak-to-peak variation is less than $40 \%$ of the average value).

Finally, the overall behavior and limited amplitude of the control torques $\tau$ in Fig. 9 indicate that the transition between free motion, collision, and hybrid force/motion task phases occur in a rather smooth way. As a result, it would be hard to recognize the collision by considering only the control torque profiles rather than the residuals.

\section{CONCLUSIONS}

Residual signals generated by a model-based fault diagnostic scheme can be used as a sensorless and efficient collision detection method for robot manipulators moving in an unstructured environment. The residual vector allows to identify the robot link that has collided and, to some extent, the contact force location and intensity. Moreover, the information content in the residual dynamics can be used for designing a hybrid force/motion controller that

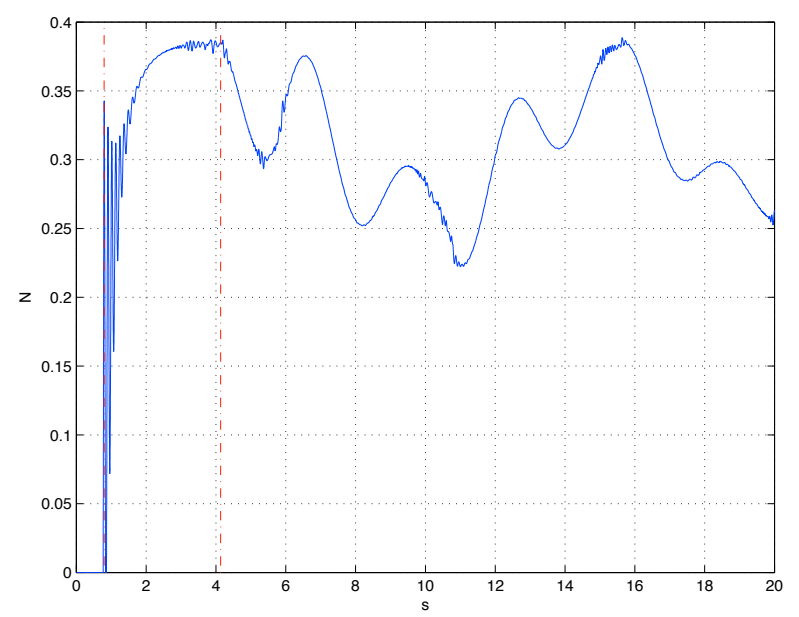

Fig. 7. Norm of the cartesian force at the contact point (normal to the obstacle)

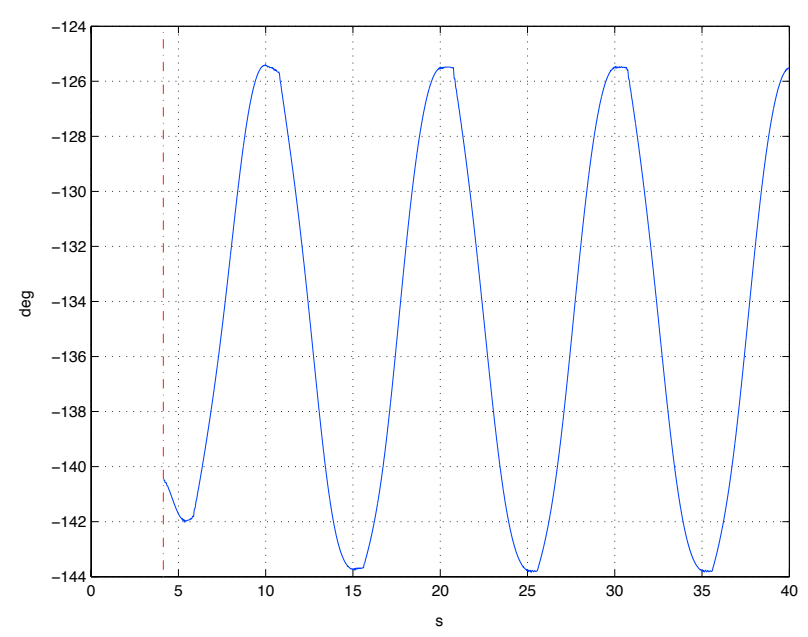

Fig. 8. Angular motion of the contact point on the obstacle contour

handles the interaction task which follows the detected collision. The novel feature of this controller is that it works on a suitably defined decomposition of joint-space variables, since the actual location and orientation of the cartesian contact frame is not known explicitly. At least for compliant obstacles, reasonable cartesian performance can still be achieved, e.g., in terms of following a trajectory on the (unknown and not sensed) obstacle surface while keeping the contact force in the neighborhood of a specified value (without direct force sensing).

The preliminary results in this paper deserve further analysis and experimental verification. On one side, it is reasonable that the backdrivability of cartesian contact forces into their joint-space counterparts (as in directdrive robots) increases the sensitivity of the sensorless collision detection method and of the associated hybrid force/motion controller. On the other hand, safety indices related to collisions are typically improved by the presence of some compliance in the robot structure. Interestingly, the proposed collision detection method can be easily extended also to the class of robots with joint transmission elasticity. 


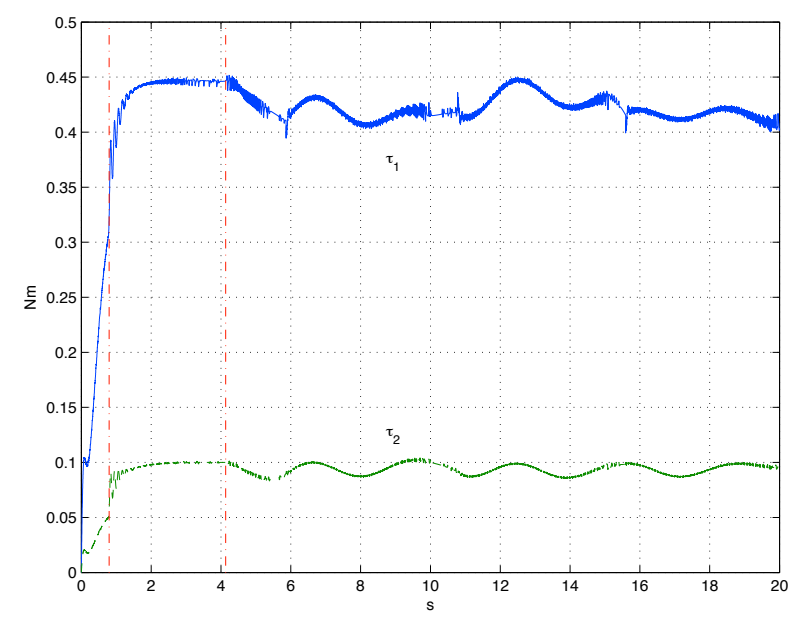

Fig. 9. Control torques

For faster collisions with stiffer environments, it is important to analyze the transient phase following the first impact. Provably stable controllers should be designed so as to bring the robot in a quasi-static condition, necessary for starting more complex (e.g., hybrid) interaction tasks.

\section{REFERENCES}

[1] J. Heinzmann and A. Zelinsky, "Quantitative safety guarantees for physical human-robot interaction," Int. J. of Robotics Research, vol. 22, no. 7/8, pp. 479-504, 2003.

[2] P. Jimenez, F. Thomas, and C. Torras, "Collision detection algorithms for motion planning," in J.-P. Laumond (Ed.), Robot Motion Planning and Control, pp. 305-343, Springer-Verlag, Berlin, 1998.

[3] V. Lumelsky and E. Cheung, "Real-time collision avoidance in teleoperated whole-sensitive robot arm manipulator," IEEE Trans. on Systems, Man, and Cybernetics, vol. 23, no. 1, pp. 194-203, 1993.

[4] D. M. Ebert and D. D. Henrich, "Safe human-robot-cooperation: Image-based collision detection for industrial robots," IEEE/RSJ Int. Conf. on Intelligent Robots and Systems, pp. 1826-1831, 2002.

[5] A. Garcia, V. Feliu, and J. A. Somolinos, "Experimental testing of a gauge based collision detection mechanism for a new three-degreeof-freedom flexible robot," J. of Robotic Systems, vol. 20, no. 6, pp. 271-284, 2003.
[6] G. Hirzinger, A. Albu-Schäffer, M. Hähnle, I. Schaefer, and N. Sporer, "On a new generation of torque controlled light-weight robots," 2001 IEEE Int. Conf. on Robotics and Automation, Seoul, KR, pp. 3356-3363, 2001.

[7] A. Bicchi and G. Tonietti, "Dealing with the safety-performance tradeoff in robot arms design and control," IEEE Robotics and Automation Mag., vol. 11, no. 2, pp. 22-33, 2004.

[8] S. Takakura, T. Murakami, and K. Ohnishi, "An approach to collision detection and recovery motion in industrial robot," 15th Annual Conf. of IEEE Industrial Electronics Society (IECON89), Boston, MA, pp. 421-426, 1989.

[9] K. Suita, Y. Yamada, N. Tsuchida, K. Imai, H. Ikeda, and N. Sugimoto, “A failure-to-safety 'Kyozon' system with simple contact detection and stop capabilities for safe human-autonomous robot coexistence," 1995 IEEE Int. Conf. on Robotics and Automation, Nagoya, J, pp. 3089-3096, 1995.

[10] Y. Yamada, Y. Hirasawa, S. Huang, Y. Uematsu, and K. Suita, "Human-robot contact in the safeguarding space," IEEE/ASME Trans. on Mechatronics, vol. 2, no. 4, pp. 230-236, 1997.

[11] K. Kosuge and T. Matsumoto, "Collision detection of manipulator based on adaptive control law," 2001 IEEE/ASME Int. Conf. on Advanced Intelligent Mechatronics, Como, I, pp. 117-122, 2001.

[12] S. Morinaga and K. Kosuge, "Collision detection system for manipulator based on adaptive impedance control law," 2003 IEEE Int. Conf. on Robotics and Automation, Taipei, ROC, pp. 1080-1085, 2003.

[13] A. De Luca and R. Mattone, "Actuator fault detection and isolation using generalized momenta," 2003 IEEE Int. Conf. on Robotics and Automation, Taipei, ROC, pp. 634-639, 2003.

[14] W. Dixon, I. Walker, D. Dawson, and J. Hartranft, "Fault detection for robot manipulators with parametric uncertainty: A predictionerror-based approach," IEEE Trans. on Robotics and Automation, vol. 16, no. 6, pp. 689-699, 2000.

[15] H.-B. Kuntze, Ch. W. Frey, K. Giesen, and G. Milighetti, "Fault tolerant supervisory control of human interactive robots," Work. on Advanced Control and Diagnosis, Duisburg, D, pp. 55-60, 2003.

[16] A. De Luca and R. Mattone, "An adapt-and-detect actuator FDI scheme for robot manipulators," 2004 IEEE Int. Conf. on Robotics and Automation, New Orleans, LA, pp. 4975-4980, 2004.

[17] S. Morinaga and K. Kosuge, "Compliant motion control of manipulator's redundant dof based on model-based collision detection system," 2004 IEEE Int. Conf. on Robotics and Automation, New Orleans, LA, pp. 5212-5217, 2004.

[18] L. Sciavicco and B. Siciliano, Modeling and Control of Robot Manipulators, 2nd Ed., Springer, London, 2000.

[19] A. De Luca and C. Manes, "Modeling robots in contact with a dynamic environment," IEEE Trans. on Robotics and Automation, vol. 10 , no. 4 , pp. 542-548, 1994. 